\title{
Behavioral Role for Nitric Oxide in Chemosensory Activation of Feeding in a Mollusc
}

\author{
Maurice R. Elphick, a György Kemenes, Kevin Staras, and Michael O'Shea \\ Sussex Centre for Neuroscience, School of Biological Sciences, University of Sussex, Brighton, BN1 9QG, \\ United Kingdom
}

A role for the NO-cGMP pathway in mediating chemosensory activation of feeding is suggested by intense NADPH diaphorase staining observed in nerve fibers that project from sensory cells in the lips to the CNS and by the presence in the CNS of a NO-activated guanylyl cyclase. In preparations reduced to isolated lips and CNS, intracellular recordings were made from motoneurons driven by the interneurons of the central pattern generator (CPG) for feeding. Fictive feeding in such preparations can be recorded from these motoneurons following the application of sucrose to the lips. Sucrose activation of fictive feeding is inhibited by the NO scavenger hemoglobin, the NO syntha-

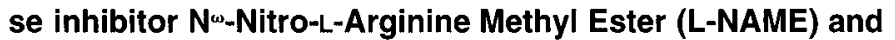
by methylene blue, an inhibitor of guanylyl cyclase. Fictive feeding in isolated lip-CNS preparations can be activated without sucrose by superfusion of NO donor molecules such as SNAP and hydroxylamine and by the nonhydrolyzable analog of CGMP, 8-bromo-cGMP. The feeding CPG can also be activated centrally by depolarizing a modulatory interneuron, the slow osciliator (SO). When the CPG is activated in this way, fictive feeding is not susceptible to inhibition by hemoglobin, the most potent of the inhibitors of sucrose-activated fictive feeding. Behavioral experiments on intact snails confirm the findings from in vitro experiments and show that hemoglobin prevents feeding and methylene blue significantly delays the onset of feeding. These results indicate (1) that NO is a putative chemosensory transmitter in the snail $L$. stagnalis, (2) that the NOcGMP pathway can mediate chemosensory activation of specific patterns of centrally generated behavior, (3) that NO is not involved in transmission within the central network of neurons responsible for the behavior, and more generally (4) that a freely diffusing and highly reactive gaseous signalling molecule can have restricted and specific behavioral functions.

[Key words: nitric oxide synthase, cyclic GMP, Lymnaea stagnalis, feeding, chemosensory, central pattern generator, NADPH diaphorase]

\footnotetext{
Received May 17, 1995; revised July 19, 1995; accepted July 27, 1995. We are very grateful to colleagues at Sussex for helpful criticism of the manuscript. This work was supported by Grant GR/J33234 from the BBSRC. Correspondence should be addressed to Prof. M. O'Shea at the above address.

"Present address: School of Biological Sciences, Queen Mary and Westfield College, University of London, Mile End Road, London, El 4NS, UK.

Copyright (C) 1995 Society for Neuroscience $0270-6474 / 95 / 157653-12 \$ 05.00 / 0$
}

The gas nitric oxide (NO) is a neuronal signalling molecule in the mammalian CNS. It is synthesized in neurons from L-arginine by a $\mathrm{Ca}^{2} /$ calmodulin-dependent NO synthase (NOS) that requires NADPH as a cosubstrate (Bredt and Snyder, 1992). Following its diffusion from sites of synthesis, NO exerts physiological effects by activating soluble guanylyl cyclase and increasing cGMP production in target cells (Bredt and Snyder, 1989; Southam and Garthwaite, 1993). While a number of physiological roles have been attributed to NO in the CNS (Schuman and Madison, 1994), it has not as yet been possible to link the cellular distribution or the actions of NO with specific behaviors. An opportunity to do so is provided by the feeding system of the freshwater snail Lymnaea stagnalis, in which many of the neuronal components generating feeding behavior are identified (for a review, see Benjamin and Elliott, 1989). This enables examination of the behavioral roles of neurotransmitters at the cellular level.

Nitric oxide synthase (NOS) is present in the Lymnaea nervous system (Elofsson et al., 1993; Elphick et al., 1994b) and we show here that NADPH-diaphorase histochemistry indicates that high levels of NOS are present in nerve fibers that project to the CNS from sensory cells in the lips. As the lips have an important function in the detection of food (Goldschmeding and Jager, 1973), we have examined the possibility that the NOcGMP signalling pathway is involved in chemosensory activation of feeding.

Feeding in the snail is accomplished by the repetition at about $0.2 \mathrm{~Hz}$ of a three-phased cycle of activity-protraction of the toothed radula, followed by a rasp, and then swallowing (Rose and Benjanin, 1979). These movements are normally provoked by the detection of nutrients and can be reliably initiated in nonfeeding snails by adding sucrose to the water (Kemenes et al., 1986). Chemosensory stimuli produce rhythmic feeding movements by activating a central pattern generator (CPG) consisting of a network of three identified classes of interneurons called N1, N2, and N3 (Rose and Benjamin, 1981b; Fig. 1A). This CPG network drives and coordinates the activity of the feeding motoneurons in the buccal ganglion. Feeding can also be influenced by central modulatory neurons that are not part of the CPG. Principal among them are the slow oscillator (SO) neuron and the serotonergic cerebral giant cells (CGCs) (McCrohan and Benjamin, 1980a,b; Rose and Benjamin, 1981a; Elliot and Benjamin, 1985b). The SO can activate the CPG without the need to stimulate the chemosensory pathway and the CGC gates and facilitates the action of the SO on the CPG (Yeoman et al., 1994a,b). Both the SO and the CGCs are excited by chemosensory stimulation of the lips (Kemenes et al., 1986). 
A

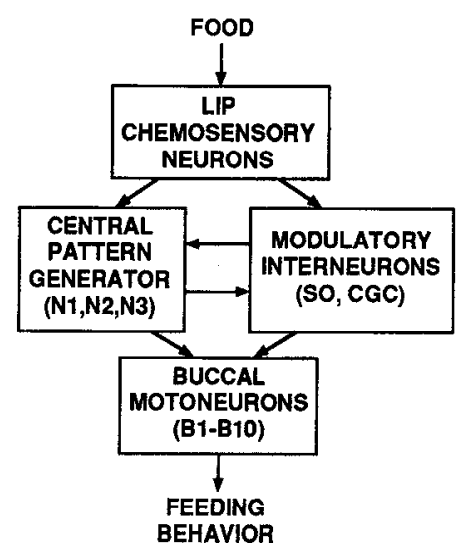

B

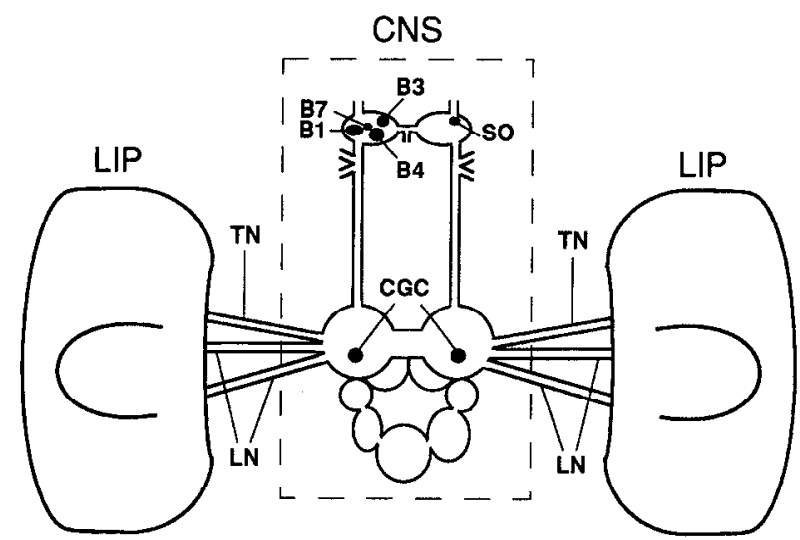

Figure 1. The feeding system of Lymnaea stagnalis. A, Model of chemosensory activation of feeding behavior in Lymnaea (based on Benjamin and Elliott, 1989). Chemosensory neurons activate a feeding central pattern generator $(C P G)$ directly and indirectly via modulatory interneurons such as the slow oscillator $(S O)$ cell and the cerebral giant cells $(C G C)$. The $\mathrm{CPG}$, comprising three classes of interneurons $(N I, N 2, N 3)$, generates rhythmic triphasic activity in buccal feeding motoneurons $(B I-B I O)$ that control radula protraction, radula retraction (rasping) and swallowing. There is feedback from the CPG to modulatory interneurons and the modulatory interneurons also form direct connections with buccal motoneurons. $B$, Diagram of the lip-CNS preparation used in electrophysiological experiments. Nerves that relay chemosensory input to the CNS, the median and superior lip nerves $(L N)$, and the tentacle nerve $(T N)$, were left intact. The cell body positions of identified neurons used in this study are illustrated. The paired feeding motoneurons $(B 1, B 3, B 4, B 7$; shown only on left) and the single slow oscillator (SO) interneuron are found in the buccal ganglia. The cell bodies of the paired cerebral giant cells $(C G C)$ are located in the cerebral ganglia. The cerebral ganglia and buccal ganglia were desheathed to provide access for microelectrodes and drugs. The sensory surfaces of the lips were kept moist with saline but were not exposed to drugs introduced into the perfusion system.

In summary, the snail feeding system consists of chemosensory activators, an identified central pattern generating circuit, modulatory interneurons that can activate the CPG independently of sensory input, and a well-defined pattern of motor activity that drives a specific behavior. Importantly, the system can be studied in preparations reduced to the isolated lips and the CNS in which intracellular recordings can be made from feeding motoneurons, elements of the feeding CPG, and modulatory neurons (Fig. 1B). In such preparations, "fictive" feeding activity can be monitored in motoneurons in response to a sucrose stimulus delivered to the lips. Fictive feeding can also be initiated by activation of the SO interneuron by intracellular current injection.

Here we have used the reduced lip-CNS preparation to investigate whether the NO-cGMP signalling pathway is involved in activating fictive feeding. The validity of the results obtained in these in vitro experiments were then tested by examining the effects of interfering with the NO-cGMP pathway in freely behaving intact animals.

\section{Materials and Methods}

Wild-type Lymnaea stagnalis were obtained from Blades Biological and fed on lettuce. All chemicals used were purchased from Sigma unless otherwise stated.

Measurement of cGMP and CAMP in the CNS. The CNS, including the cerebral and buccal ganglia, was removed from specimens of Lymnae a and incubated individually at room temperature with the NO donors hydroxylamine ( $1 \mathrm{mM}$ ) or $S$-nitroso- $N$-acetylpenicillamine (SNAP, $0.5 \mathrm{~mm}$; purchased from Calbiochem-Novabiochem (UK) Ltd., Nottingham) dissolved in $200 \mu \mathrm{l}$ HEPES buffered Lymnaea physiological saline (Benjamin and Winlow, 1981). It is well documented that both SNAP and hydroxylamine liberate NO under physiological conditions (Feelisch, 1991) and both are widely used as sources of NO in biochemical and physiological experiments.

Control incubations were performed in physiological saline alone. At time zero and 5 and $10 \mathrm{~min}$ of incubation cyclic nucleotides were extracted from the CNS by boiling $(5 \mathrm{~min})$ in $400 \mu \mathrm{l} 50 \mathrm{~mm}$ sodium acetate $(\mathrm{pH} 4.75)$ followed by homogenization with a glass pestle. Ex- tracts were centrifuged for $5 \mathrm{~min}$ in a microfuge and the cAMP, and cGMP content of the supernatant was measured using radioimmunoassays, as described previously (Elphick et al., 1993).

NADPH diaphorase histochemistry. Tissue was fixed with $4 \%$ paraformaldehyde in phosphate-buffered saline (PBS) at $4^{\circ} \mathrm{C}$ for $4 \mathrm{hr}$ and then cryo-protected in $10 \%$ sucrose/PBS overnight at $4^{\circ} \mathrm{C}$. After embedding in Tissue-Tek O.C.T. Compound (Miles Inc., USA), serial 30 $\mu \mathrm{m}$ sections were cut using a Leica CM 3000 cryostat and collected on chrome-alum/gelatin-coated slides. Slides were washed in $50 \mathrm{~mm}$ Tris$\mathrm{HCl}(\mathrm{pH} 7.5)$ and then incubated in $1 \mathrm{~mm}$ NADPH/0.25 mM nitro blue tetrazolium in Tris- $\mathrm{HCl}$ at room temperature (in the dark) for about 1 hr. After washing in water, sections were mounted with an aqueous mounting medium (Immun-mount, Shandon).

Electrophysiological experiments on lip-CNS preparations. The semi-intact lip-CNS preparation consisted of the lips and tentacles separated into two halves by cutting down the midline and removing most of the foot (Kemenes et al., 1986; Fig. 1B). The lip and tentacles were connected to the cerebral ganglia via the left and right median and superior lip nerves and the tentacle nerves. All other peripheral nerves were cut. The buccal ganglia were left connected to the cerebral ganglia via the paired cerebro-buccal connectives (Fig. 1B). Thus, the only pathway from the lips to the buccal ganglia was via the lip and tentacle nerves and through the cerebral ganglia and the cerebro-buccal connectives. Sucrose $(0.1 \mathrm{M})$ was added to both lip-tentacles by simply placing a drop of solution onto the sensory surfaces kept moist with saline.

Intracellular recordings were made simultaneously from three neurons while the preparation was maintained in HEPES buffered Lymnaea physiological saline. Electrodes were drawn from $2 \mathrm{~mm}$ capillary tubing (Clark Electromedical) and filled with $4 \mathrm{~m}$ potassium acetate, giving tip resistances of 30-80 $\mathrm{M} \Omega$ Signals were amplified with Neurolog amplifiers and fed to an oscilloscope, a DAT recorder (Biologic) and a chart recorder (Gould). Recordings were made from four different types of buccal motoneuron (B1, B3, B4, B7) and a modulatory interneuron in the cerebral ganglia (the CGC) for several minutes, before, during, and after the application of sucrose to the lips. Normally, in this preparation, application of sucrose to the lips causes an increase in the tonic firing frequency of the CGCs and activation of the feeding CPG, reflected in the rhythmic activity of buccal motoneurons (Kemenes et al., 1986, and Fig. 4A)

In experiments designed to examine the effect of interference with the NO-cGMP pathway on sucrose activation of fictive feeding, the CNS was bathed for 10 min with reagents known to inhibit the pathway. 
In other experiments, reagents known to activate the pathway were introduced into the perfusion system. In each experiment the competence of the preparation to respond to sucrose was first established.

Hemoglobin ( $\mathrm{Hb}$ ), which binds $\mathrm{NO}$, was tested at a concentration $(0.2 \mathrm{~mm})$ similar to that used in previously reported neurophysiological studies on NO (Pape and Mager, 1992). Met-hemoglobin, which does not bind NO, was used to control for the effects of hemoglobin that are not related to its ability to scavenge NO. It was prepared by incubating $1 \mathrm{gm}$ of bovine hemoglobin dissolved in $0.01 \mathrm{M}$ potassium phosphate (pH 7.7) with $10 \mathrm{~mm}$ sodium nitrite at room temperature for about $1 \mathrm{hr}$ and then dialyzing against 1.5 liters of distilled water overnight at $4^{\circ} \mathrm{C}$ (method based on Moriguchi et al., 1992).

The L-arginine analog $\mathrm{N}^{\omega}$-Nitro-L-arginine Methyl Ester (L-NAME) is one of several specific inhibitors of NOS (Moncada et al., 1991). It was used at $10 \mathrm{~mm}$, a concentration selected because it causes total inhibition of NOS activity in vitro (Lambert et al., 1990). Also, this concentration has been used in other electrophysiological studies on NO action in the molluscan CNS (Gelperin, 1994). D-NAME was used as a control for L-NAME at the same concentration.

In a related set of experiments we tested if $\mathrm{L}$-arginine could displace L-NAME and reverse the inhibition of the NO-cGMP pathway and its effect on the activation of feeding. This displacement test was based on similar experiments used to assess the role of NO in network oscillations in the olfactory system of another mollusc, Limax (Gelperin, 1994). Snails $(n=6)$ were first tested for sucrose response with the brain in normal HEPES. After a 10 min perfusion of the brain with 10 nM L-NAME, the snails were again tested with sucrose applied to the lips. This was followed by a $10 \mathrm{~min}$ wash with a mixture of $10 \mathrm{~mm}$ L-NAME and $5 \mathrm{~mm}$ L-arginine. The snails were then retested for the sucrose response. Mean responses (fictive feeding cycles per minute) in HEPES, L-NAME and L-NAME plus L-arginine were compared using two-tailed $t$ tests.

Methylene blue (MB), which is widely recognised as an inhibitor of the NO target enzyme soluble guanylyl cyclase (Miki et al., 1987), but which may also affect NO-cGMP signalling through inhibition of $\mathrm{NO}$ synthesis by NOS (Mayer et al., 1993), was used at $0.2 \mathrm{~mm}$. This concentration was selected because it has been shown to cause complete inhibition of guanylyl cyclase activity (Mayer et al., 1993).

The effects of these drugs on fictive feeding were evaluated by counting the number of fictive feeding cycles for the $1 \mathrm{~min}$ period immediately before and immediately following the application of sucrose to the lips. All preparations used were quiescent or spontaneously active at a slow rate prior to sucrose treatment.

Stimulation of the NO-cGMP signalling pathway was achieved by the application of the NO donors $S$-nitroso- $N$-acetylpenicillamine (SNAP, $1 \mathrm{mM}$ ) and hydroxylamine ( $1 \mathrm{mM}$ ), and the cGMP analog 8-Bromo-cGMP $(3 \mathrm{~mm})$. Both NO donors were used in physiological experiments at $1 \mathrm{~mm}$ because at this they cause significant stimulation of cGMP synthesis in the Lymnaea CNS (unpublished data and Fig. 2).

Fictive feeding was activated without providing a sucrose stimulus to the lips by direct activation of the modulatory interneuron SO by injecting it with depolarizing current. This allowed us to investigate whether the NO-cGMP signalling pathway is involved in patterned activity generated by the feeding CPG in the absence of sensory inputs.

Behavioral experiments. Experiments comparing the effects of hemoglobin or Met-hemoglobin injected into intact animals were carried out by two of us. Injections were performed by one of us without knowing whether the solution contained hemoglobin or Met-hemoglobin and the behavioral testing was performed by another on numbered animals The experiments on methylene blue injected animals could not be performed blind because injected snails are conspicuously blue!

When hungry snails are transferred from water to a sucrose-rich environment they emerge from their shells and feeding usually starts within $30 \mathrm{sec}$ (Kemenes et al., 1986). We examined whether this feeding response is affectcd by inhibitors of the NO-cGMP pathway by inject ing groups of 12 hungry snails with $200 \mu$ of hemoglobin $(1 \mathrm{~mm})$ or methylene blue $(1 \mathrm{~mm})$ or their respective controls $(200 \mu \mathrm{l}$ of $1 \mathrm{~mm}$ Met-hemoglobin and $200 \mu \mathrm{l}$ of water). The drug concentrations and injected volumes were selected to give final concentrations in the circulation similar to those used in the in vitro experiments with semiintact lip-CNS preparations ( $0.2 \mathrm{mM})$.

After $3 \mathrm{hr}$, to allow for recovery from the injection, individual snails were placed in water containing $10 \mathrm{~mm}$ sucrose and their behavior observed and scored. A sucrose concentration of $10 \mathrm{~mm}$ was used because the sensitivity of intact animals to sucrose is about one order of mag-

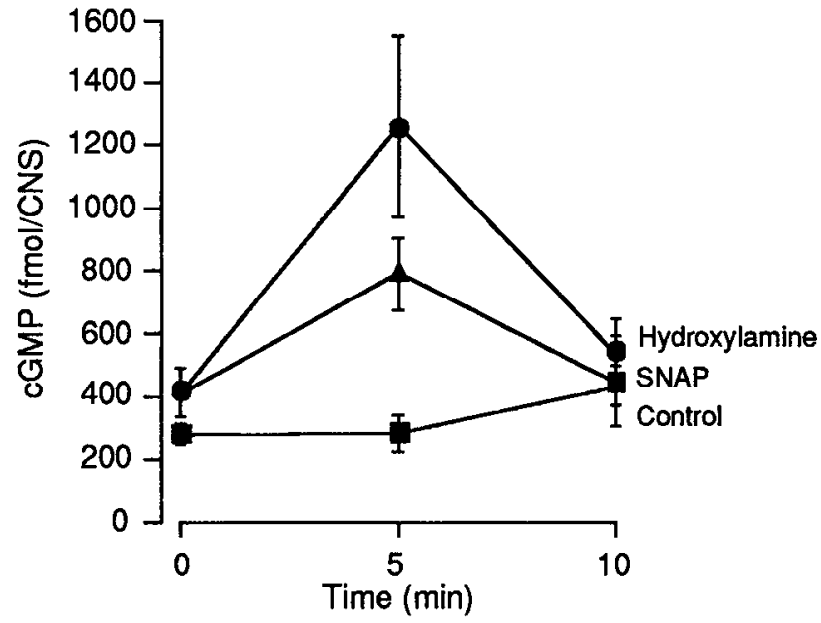

Figure 2. NO donors activate cGMP synthesis in Lymnaea CNS. Hydroxylamine $(1 \mathrm{~mm})$ and $S$-nitroso- $N$-acetyl penicillamine $(S N A P, 0.5$ mM) both caused a transient increase in the mean cGMP content of the CNS (error bars, SEM; $n=3$ ) compared with control (HEPES physiological saline). The cAMP content of the CNS was unaffected by treatment with NO donors (not shown).

nitude greater than that of the semi-intact preparations (Kemenes et al., 1986). For each animal we measured the time taken to emerge from its shell and counted the number of rasps for 2 min after emergence. This was achieved using a custom written computer program (SnailTrace), which acts as a sophisticated event recorder. The software records the duration and timing of a variety of behaviors associated with feeding and then generates statistics using the data collected. For each subgroup of four animals the number of rasps occurring in each $10 \mathrm{sec}$ interval of the observation period was counted. These numbers were used to calculate means and standard errors at each interval for the whole group of 12 animals. Where appropriate, a two-factor ANOVA with replication was performed, and if this revealed a source of significant differ ences, pair-wise comparisons were made using two-tailed $t$ tests.

\section{Results}

The NO-cGMP signalling pathway

When the isolated Lymnaea CNS was incubated with the compounds SNAP and hydroxylamine there was a transient increase above control levels in its cGMP content (Fig. 2). Under the same conditions, levels of cAMP remain unchanged (not shown). NO donor-induced cGMP production in the Lymnaea CNS demonstrates the presence of a NO-activated guanylyl cyclase. This observation is consistent with previous findings in both vertebrates (Southam and Garthwaite, 1991) and insects (Elphick et al., 1993). Coupled with previously reported evidence (Elofsson et al., 1993; Elphick ct al., 1994b) for the presence of a $\mathrm{Ca}^{2+} /$ calmodulin-dependent NOS, these experiments show that the two principal enzymes of the NO-cGMP signalling pathway are present in the snail CNS.

In order to determine whether a neuronal NOS is anatomically associated with the feeding system in Lymnaea, we have used the NADPH diaphorase staining method. In mammals, this technique has been shown to be a reliable histochemical indicator of NOS and NADPH-diaphorase staining is generally accepted as evidence for the localization of NOS in the nervous system (Dawson et al., 1991; Hope et al., 1991). Moreover, in insects, neuronal NOS does not differ from its mammalian counterpart with respect to cofactor and cosubstrate dependence, and the distribution of NADPH diaphorase staining can be accounted for by the distribution of NOS activity (Elphick et al., 1993, 1994a, 1995). It seems likely therefore that the NADPH-dia- 
phorase technique is a valid indicator of NOS in the Lymnaea nervous system. Moreover, we also provide further biochemical and physiological evidence supporting our conclusions based on this method.

NADPH diaphorase histochemistry reveals intense staining of a large population of nerve fibers in the median and superior lip nerves and the tentacle nerves (Fig. $3 A, B$ ) that project into the neuropile of the cerebral ganglia. Similar intense staining is present in the inferior, median, and superior pedal nerves (not shown), which innervate the foot. In contrast, nerves innervating visceral organs such as the genital nerve and intestinal nerve contain little or no staining (Fig. 3C). Thus, NADPH diaphorase staining appears to be associated with nerves carrying fibers from sensory cells in the skin to the CNS. This interpretation is supported by the absence of NADPH diaphorase stained neuronal somata in the circumoesophageal ganglia of the CNS (Fig. $3 A-C)$. The technique does, however, reveal a small population of stained cell bodies in the buccal ganglia, which have been described previously by ourselves and by others (Elofsson et al., 1993; Moroz et al., 1993; Elphick et al., 1994b).

The intense staining of the lip nerves is interesting because these nerves are involved in chemosensory activation of feeding behavior in Lymnaea (Goldschmeding and Jager, 1973). It suggests that NOS may be present in chemosensory neurons of the lips and that NO may play a role in initiating feeding behavior. Histochemical analysis of the lips revealed NADPH diaphorase stained bipolar cells with axons projecting into distal branches of the lip nerves (Fig. 3D). These cells closely resemble the lip sensory neurons described by Zaitseva and Bocharova (1981). Cells of this type are especially abundant at the ventral margins of the lips, and it is likely that the axons of these cells that are responsible for the dense staining seen in the lip nerves and in the neuropile of the cerebral ganglia. Modulatory interneurons in the Lymnaea feeding system, such as the CGCs, arborize in the regions of the cerebral ganglia occupied by NADPH diaphorase positive fibers from the lips and it is likely that this is where they receive chemosensory input. The anatomy and projection of NADPH diaphorase positive cells in the lip is therefore entirely consistent with that expected for lip chemosensory neurons involved in activation of feeding in Lymnaea. Moreover, this result suggested that the NO-cGMP pathway mediates chemosensory activation of feeding.

Sucrose-induced fictive feeding is inhibited by inhibiting the NO-cGMP pathway

When sucrose is applied to the lips of the lip-CNS preparation, in addition to a tonic depolarization, a pattern of rhythmic spiking or rhythmic subthreshold activity can be elicited in motoneurons of the buccal ganglion. The relationship between this sucrose-activated central motor pattern and the phases of the feeding cycle have been confirmed by combined electromyographic and cinephotographic recording of the buccal muscles (Rose and Benjamin, 1979). In the present study, activity patterns were recorded intracellularly from buccal motoneurons B1, $\mathrm{B} 3, \mathrm{~B} 4$, and B7. Previous studies have shown that motoneurons $\mathrm{B} 1$ and $\mathrm{B} 7$ are active during the protraction phase, B3 is active throughout the rasping phase, and B4 is active from midrasping to midswallowing phase (Benjamin and Rose, 1979; Rose and Benjamin, 1979). Activation of patterned activity in these motoneurons with the features, phase relationships, and approximate trequency characteristic of feeding behavior occurred in over two-thirds of the preparations tested $(n=26)$. In these responsive preparations activation of fictive feeding could be repeated at least three times without deterioration of the response. In the responsive preparations, fictive feeding was initiated almost immediately following the sucrose stimulus and continued without interruption for the duration of the $1 \mathrm{~min}$ poststimulus observation period. While the overall pattern of activity induced by sucrose is indicative of the activation of the feeding $\mathrm{CPG}$, the frequency of fictive feeding in the lip-CNS preparation is slightly lower than the frequency of biting during feeding behavior in whole animals. Thus, during the first minute after application of sucrose to the lips in the lip-CNS preparation there were $10 \pm 2.3$ SEM fictive feeding cycles compared with 12-14 rasps carried out by intact animals during the first minute of feeding in the presence of $10 \mathrm{~mm}$ sucrose (Kemenes et al., 1986). Nevertheless, we believe the rhythmic spiking and subthreshold activity to be true fictive feeding, indicating that the feeding CPG has been activated. Additional evidence that this activity represents a true fictive behavior is provided by the observation that quinine stops sucrose-initiated feeding in intact animals and also stops the sucrose-initiated patterned motor activity in the lip-CNS preparation (Kemenes et al., 1986). We were confident therefore that sucrose activates the feeding CPG network in the lip-CNS preparation and that we can therefore use this to investigate the possible role of the NO-cGMP signalling pathway in mediating the chemosensory activation of feeding.

To test the effect of inhibitors of the NO-cGMP signalling pathway on fictive feeding the lip-CNS preparation was first tested for responsiveness to sucrose under control conditions where the CNS is bathed in physiological saline. In responsive preparations, application of $0.1 \mathrm{M}$ sucrose to the lips caused an increase in the rate of spiking in the $\mathrm{CGC}$ interneuron and activation of the CPG, which resulted in rhythmic spiking or subthreshold rhythmic synaptic activity in buccal motoneurons. Figure $4 A$ shows the activation by sucrose of a modulatory CGC interneuron followed by strong excitation and rhythmic spiking in the B1 motoneuron and rhythmic subthreshold activity in the B4 motoneuron. Motoneuron activity in the lip-CNS preparation in response to sucrose can vary between individuals, and excitation by the CPG does not always reach threshold for spiking in some of the motoneurons. Nevertheless, the pattern of the subthreshold synaptic input can be identified as being derived from premotor interneurons of the well-characterized feeding CPG circuit (Rose and Benjamin, 1981b; Elliott and Benjamin, 1985a; Kemenes and Elliott, 1994).

When the responsive preparation illustrated in Figure $4 A$ was bathed for $10 \mathrm{~min}$ with the NO-scavenger hemoglobin ( $\mathrm{Hb}, 0.2$ $\mathrm{mM}$ ), sucrose-induced fictive feeding was completely suppressed (Fig. 4B), although the modulatory CGC interneuron was still weakly excited. Complete suppression of responsiveness by hemoglobin occurred in 3 out of 12 preparations tested and was reversible (Fig. 4C). All preparations, however, showed that hemoglobin inhibits the activation of fictive feeding by sucrose, and a summary of these data is shown in Figure $5 A$ in which the effects of hemoglobin and Met-hemoglobin are compared. Met-hemoglobin, which cannot act as an NO scavenger, allowed us to control for possible nonspecific actions of hemoglobin. In the Met-hemoglobin control, sucrose activated a mean of $6 \pm$ 1.4 SE $(n=4)$ cycles of fictive feeding during the first minute following sucrose application compared to $2.3 \pm 1.6 \mathrm{SE}(n=$ 12 ) in the presence of hemoglobin. This difference is significant ( $p<0.001, t$ test), and shows that the inhibition of feeding can 

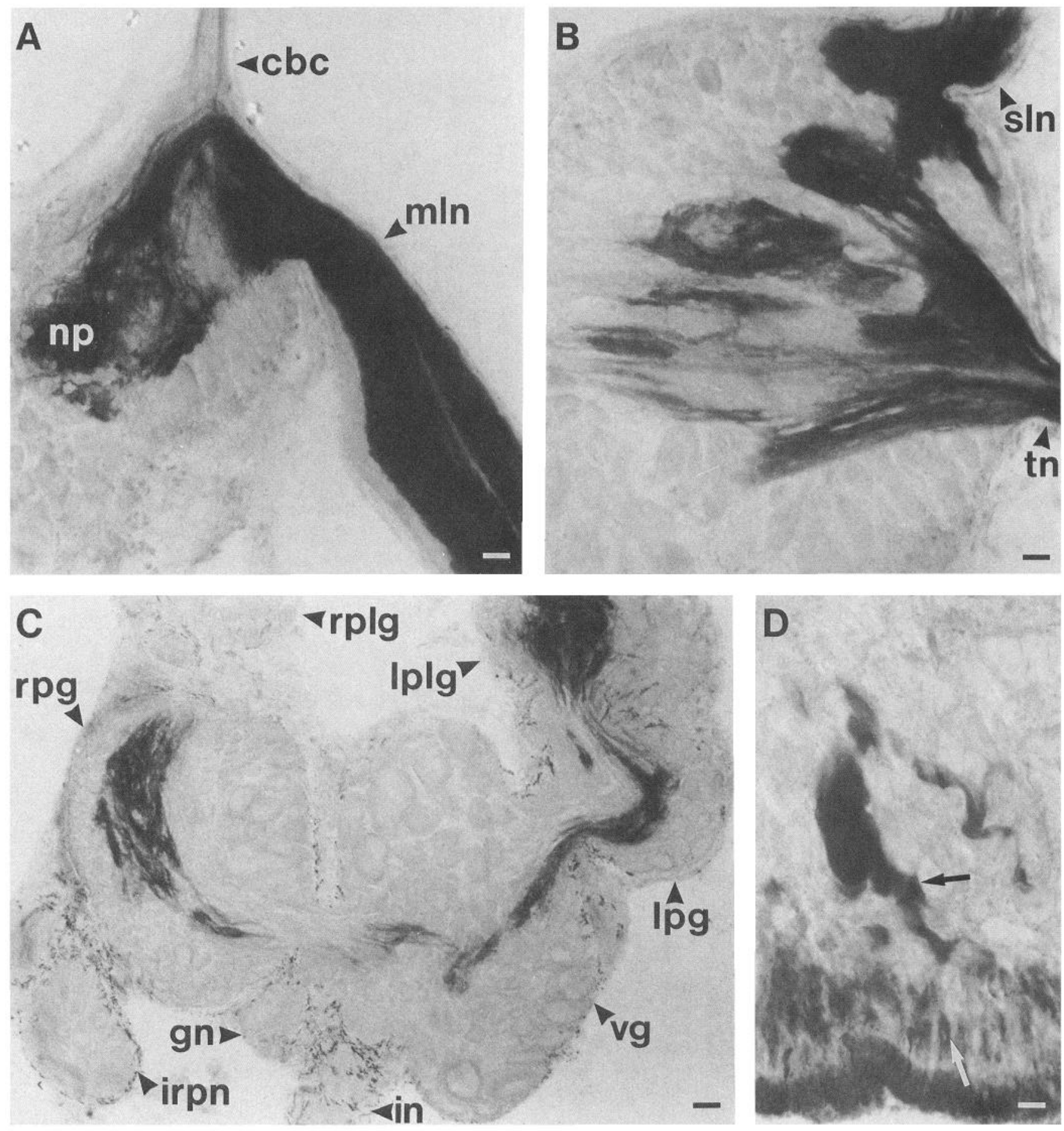

Figure 3. Localization of NADPH diaphorase. $A$ and $B$, Intense NADPH diaphorase staining is present in a large population of nerve fibers that project into the neuropile $(n p)$ of the cerebral ganglion from the median lip nerve $(m l n)$, the superior lip nerve $(s l n)$, and tentacle nerve $(t n)$. Note the absence of stained neuronal cell bodies in the cortex of the cerebral ganglia. Scale bar, $25 \mu \mathrm{m}$. $C$, NADPH diaphorase stained fibers are not apparent in visceral nerves such as the genital nerve ( $g n)$ and intestinal nerve (in). Staining is, however, present in the nerve tract that links the subesophageal ganglia of the CNS [right and left pleural $(r p l g, l p l g)$ and parietal $(r p g, l p g)$ ganglia and visceral ganglion ( $v g)$ ]. Fibers that contribute to this tract are derived from the cerebral nerve inputs (see $A$ and $B$ ) and from the parietal nerves (e.g., inner right parietal nerve, irpn). As in the cerebral ganglia $(A$ and $B)$, note the absence of stained neuronal cell bodies. Scale bar, $50 \mu \mathrm{m}$. $D$, NADPH diaphorase-stained subepithelial cells are located at the ventral margin of the upper lips. Some of these cells are bipolar neurons (white arrow) similar to sensory neurons previously described in Lymnaea (Zaitseva and Bocharova, 1981). There are also heavily stained distal branches of the lip nerves (black arrow) in the region. Scale bar, $25 \mu \mathrm{m}$. 


\section{A cNS in HEPES}

Figure 4. The NO-scavenger hemoglobin inhibits sucrose-induced fictive feeding. This figure shows responses recorded in a single preparation and $A-$ $C$ show sequential responses before hemoglobin treatment $(A)$, after hemoglobin treatment $(B)$, and after washing (C). $A$, When the CNS is bathed in HEPES physiological saline, application of sucrose $(0.1 \mathrm{M})$ to the lips evokes fictive feeding activity. The modulatory CGC interneuron is tonically excited and the buccal motoneurons $(B 1, B 4)$ are depolarized and phasically activated. The tonic depolarization of the buccal motoneurons is caused by excitatory input from the CGC cells. Rhythmic activity in the buccal motoneurons is generated by the feeding central pattern generator $(C P G)$, which is activated by chemosensory input. In this preparation, CPG input elicits rhythmic spiking in $B 1$ and subthreshold rhythmic activity in B4. $B$, When the CNS is bathed in hemoglobin $(0.2 \mathrm{mM})$, the tonic response to sucrose is almost completely blocked in the CGC and activation of rhythmic fictive feeding in buccal motoneurons is abolished. $C$, The inhibitory effects of hemoglobin are reversed by washing $(10 \mathrm{~min})$ of the preparation.

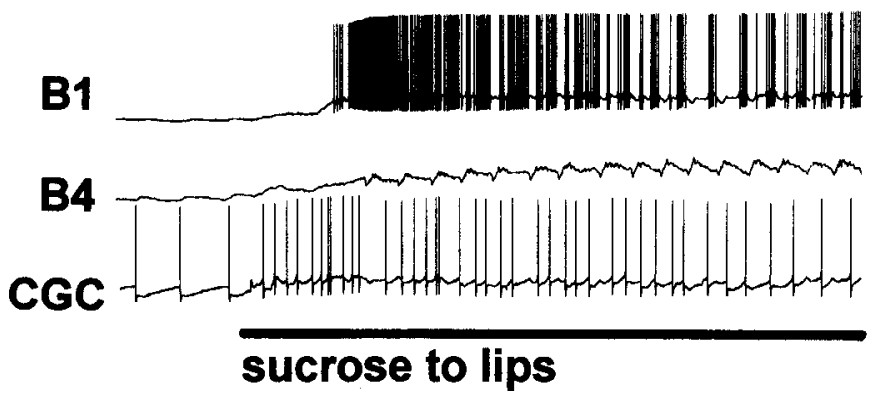

B cNS in $\mathrm{Hb}$

B1

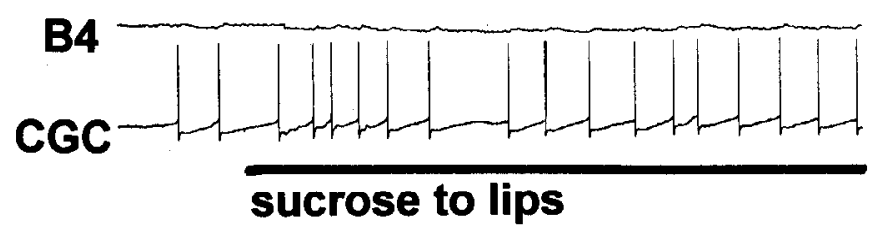

C cNS washed in HEPES
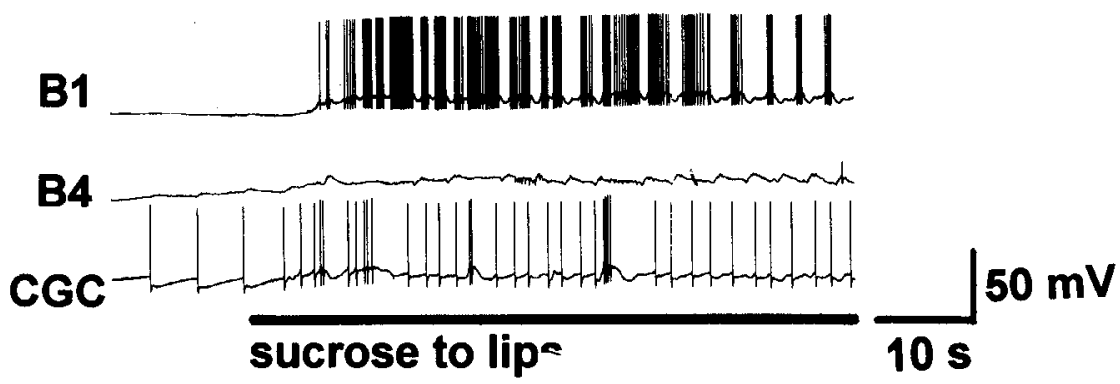

be attributed to NO scavenging by hemoglobin. The mean frequency of sucrose-activated fictive feeding was higher in $\mathrm{HE}$ PES-buffered physiological saline $(10 \pm 2.3 \mathrm{SE})$ than in the presence of Met-hemoglobin, but this difference was not significant (compare Fig. $5 \mathrm{~A}$ with $\mathrm{C}$ ). If there is weak inhibition by Met-hemoglobin, it could be attributed to the nonspecific effects of a large protein molecule at a high concentration $(0.2 \mathrm{~mm})$ or perhaps to the presence of residual hemoglobin in the Met-hemoglobin solution.

When sucrose-responsive lip-CNS preparations were bathed with $10 \mathrm{mM}$ of the NOS inhibitor L-NAME for $10 \mathrm{~min}$, sucrose activation of fictive feeding was inhibited. This inhibition is specific to the L-isomer of NAME (Fig. $5 B$ ). Sucrose activates a mean of $3.2 \pm 0.6 \mathrm{SE}(n=5)$ fictive feeding cycles in the first minute after stimulation in the presence of L-NAME and $8.8 \pm$ $0.4 \mathrm{SE}(n=5)$ in the presence of D-NAME ( $p<0.01, t$ test).

In order to show that the NOS inhibitor was acting by preventing the metabolism of arginine to NO, we performed experiments in which L-NAME and L-arginine were provided together. As expected, $10 \mathrm{mM}$ L-NAME caused a significant inhibition of feeding response in all the animals tested in this experiment ( $n=6, p<0.02$ ). The mean fictive feeding rate in HEPES was $8.8 \pm 0.8 \mathrm{SE}$ cycles $/ \mathrm{min}$, and this dropped to 1.8 $\pm 0.8 \mathrm{SE}$ cycles/min in $10 \mathrm{mM} \mathrm{L-NAME}$. In four out of the six snails this inhibition was completely reversed after a $10 \mathrm{~min}$ wash with a mixture of $10 \mathrm{mM}$ L-NAME and $5 \mathrm{~mm}$ L-arginine in HEPES. In two animals, the reversal was incomplete. Using data from all six animals for the calculations, the mean fictive feeding rate in the L-NAME plus L-arginine mixture was $6.2 \pm$ 1.4 SE, significantly higher than in L-NAME alone $(p<0.02)$ and not significantly different from feeding rates in HEPES ( $p$ $=0.13$ ).

Further evidence for a role of the NO-cGMP pathway in feeding is provided by the effect of methylene blue, an inhibitor of the soluble guanylyl cyclase (Miki et al., 1987), on sucroseactivated fictive feeding. When a sucrose-responsive lip-CNS preparation is bathed in $0.2 \mathrm{mM}$ methylene blue, sucrose-induced fictive feeding is completely or partially inhibited $(n=6)$. A summary of the data related to the effect of methylene blue is presented in Figure $5 C$, which shows that sucrose activates a mean of $1.7 \pm 0.4 \mathrm{SE}$ cycles of fictive feeding compared with $10 \pm 2.3 \mathrm{SE}$ in physiological saline $(p<0.01)$

Fictive feeding is activated by NO-donors and by 8-bromo-cGMP Application of the NO-donor SNAP to the CNS caused an increase in the spiking frequency of the CGCs and initiated the 


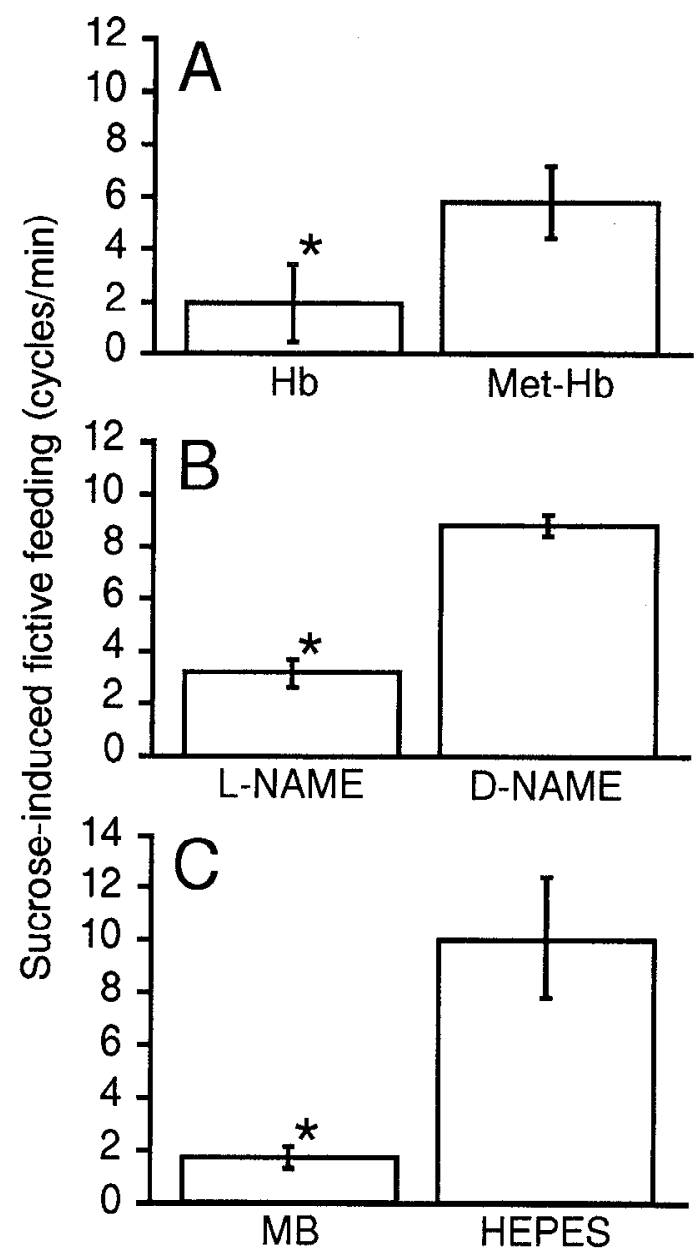

Figure 5. Effect of NO-cGMP pathway inhibitors on sucrose-induced fictive feeding. The graphs show the mean ( \pm SEM) number of fictive feeding cycles in buccal motoneurons during a one minute period following application of sucrose to the lips. A, Comparison of the effect of the NO scavenger hemoglobin $(H b ; 0.2 \mathrm{mM} ; n=12)$ with Methemoglobin (Met-Hb;0.2 mM;n=4), which does not bind NO. B, Comparison of the effect of the NO synthase inhibitor $\mathrm{N}^{\omega}-\mathrm{Nitro}-\mathrm{L}-\mathrm{Ar}-$ ginine Methyl Ester (L-NAME; $10 \mathrm{mM} n=5$ ) with its D-isomer D-NAME $(10 \mathrm{mM} ; n=5)$. $C$, Comparison of the effect of the soluble guanylyl cyclase inhibitor methylene blue $(M B ; 0.2 \mathrm{mM} ; n=6)$ with HEPES physiological saline $(n=18)$. All three NO-cGMP pathway inhibitors caused a significant $(* p<0.01, t$ test, $n=4-18)$ reduction in the number of fictive feeding cycles during the first minute after application of sucrose to the lips compared to controls. The inhibitory effect of $\mathrm{Hb}$ was reversed by washing (Fig. 4C), but the inhibitory effects of L-NAME and MB were not reversed after a 10 min wash.

characteristic CPG-driven fictive feeding activity in buccal motoneurons $(n=5)$ (Figs. 6A, 7B). Another NO donor, hydroxylamine, had similar but delayed effects $(n=5)$. Thus, hydroxylamine caused delayed tonic excitation of the CGCs and a delayed rhythmic activation of the motoneurons (Figs. 6B, 7C). This difference in the time course of action of these two NO donors is consistent with mechanisms by which they release NO. SNAP spontaneously generates NO once in solution, whereas hydroxylamine is metabolized to liberate NO by cellular enzymes such as catalases (Southam and Garthwaite, 1991).

Application of the membrane permeable cGMP analog 8-bromo-cGMP $(n=7)$ also caused delayed tonic excitation of the $\mathrm{CGCs}$ and delayed activation of the CPG-driven fictive feeding pattern in the motoneurons (Figs. $6 C, 7 D$ ). Note that similarly to sucrose given to the lips (Fig. $4 A, C$, and Kemenes et al, 1986), all these drugs also cause a general depolarization of the feeding motoneurons and the CGC when added to the CNS. The specific feeding CPG-driven rhythmic activity is superimposed on this general depolarization (Figs. $4 A, C, 6$ ), and this specific effect is analyzed in detail in Figure 7 . For this analysis we counted the number of fictive feeding cycles in 1 min intervals for the $2 \mathrm{~min}$ before and $5 \mathrm{~min}$ after the application of HEPES, SNAP, HA, or 8-Br-cGMP to the preparation.

\section{Centrally activated fictive feeding is not inhibited by inhibiting the NO-cGMP pathway}

Fictive feeding can be activated without stimulating the lips by exciting the SO modulatory interneuron (Rose and Benjamin, 1981a; Elliott and Benjamin, 1985b). Figure $8 A$ shows the effect of a $50 \mathrm{sec}$ suprathreshold depolarization of the SO on the activity of the B7 and B4 motoneurons. Typically, such prolonged depolarization produces fictive feeding activity with a frequency ranging from 9 to 15 cycles/min $(n=6)$.

In order to examine whether the NO-cGMP pathway is involved in the central organization or activation of feeding, we tested the effect of hemoglobin on the activation of fictive feeding by the SO interneuron (Fig. $8 B$ ). Hemoglobin has a potent and reversible inhibitory effect on sucrose-induced fictive feeding but has no effect on SO-driven fictive feeding. Hemoglobin had no effect on the pattern of activity activated centrally; neither did it significantly alter the frequency of SO-activated feeding cycles $(p=0.95, n=6)$. These results indicate that NO is required for the activation of feeding through the sensory pathway but is not required for transmission within the central network of neurons that control and generate feeding.

\section{Experiments on intact animals}

In these experiments we investigated the behavioral effects of injecting intact animals with hemoglobin, Met-hemoglobin, methylene blue, and water. Our experiments using the lip-CNS preparation suggested that inhibition of the NO-cGMP pathway by hemoglobin and methylene blue would specifically suppress the behavioral response to sucrose in intact animals. In order to interpret the results in this way, however, it is important to show that the injection itself did not have a nonspecific inhibitory or depressing effect on the animals' behavior. When untreated animals are transferred to a test dish containing a sucrose they first withdraw into their shells but then emerge to start locomotion and rasping within about $30 \sec (25 \pm 4.3 \mathrm{SEM})$. When animals are allowed a $3 \mathrm{hr}$ recovery period following injection with 200 $\mu \mathrm{l}$ of water or $200 \mu \mathrm{l}$ of hemoglobin, Met-hemoglobin, or methylene blue solutions, they also withdraw when being transferred to the test dish. There was, however, no significant difference in the emergence times of untreated and injected animals (ANOVA $p=0.56$ ), and all the snails displayed normal locomotory behavior after emergence. These observations indicated that the general spontaneous behavior of the animals was not affected by treatment with the two inhibitors of the NO-cGMP pathway.

While appearing to be behaviorally normal, animals injected with inhibitors of the NO-cGMP pathway differed markedly from control animals in their response to sucrose (Fig. 9). All individuals in the untreated control group (not shown) and the water injected control group started feeding within a 2 min test period following emergence in sucrose-rich water. In both of these control groups a maximal rasping rate was observed during the first $10 \mathrm{sec}$ after emergence and was sustained for the du- 

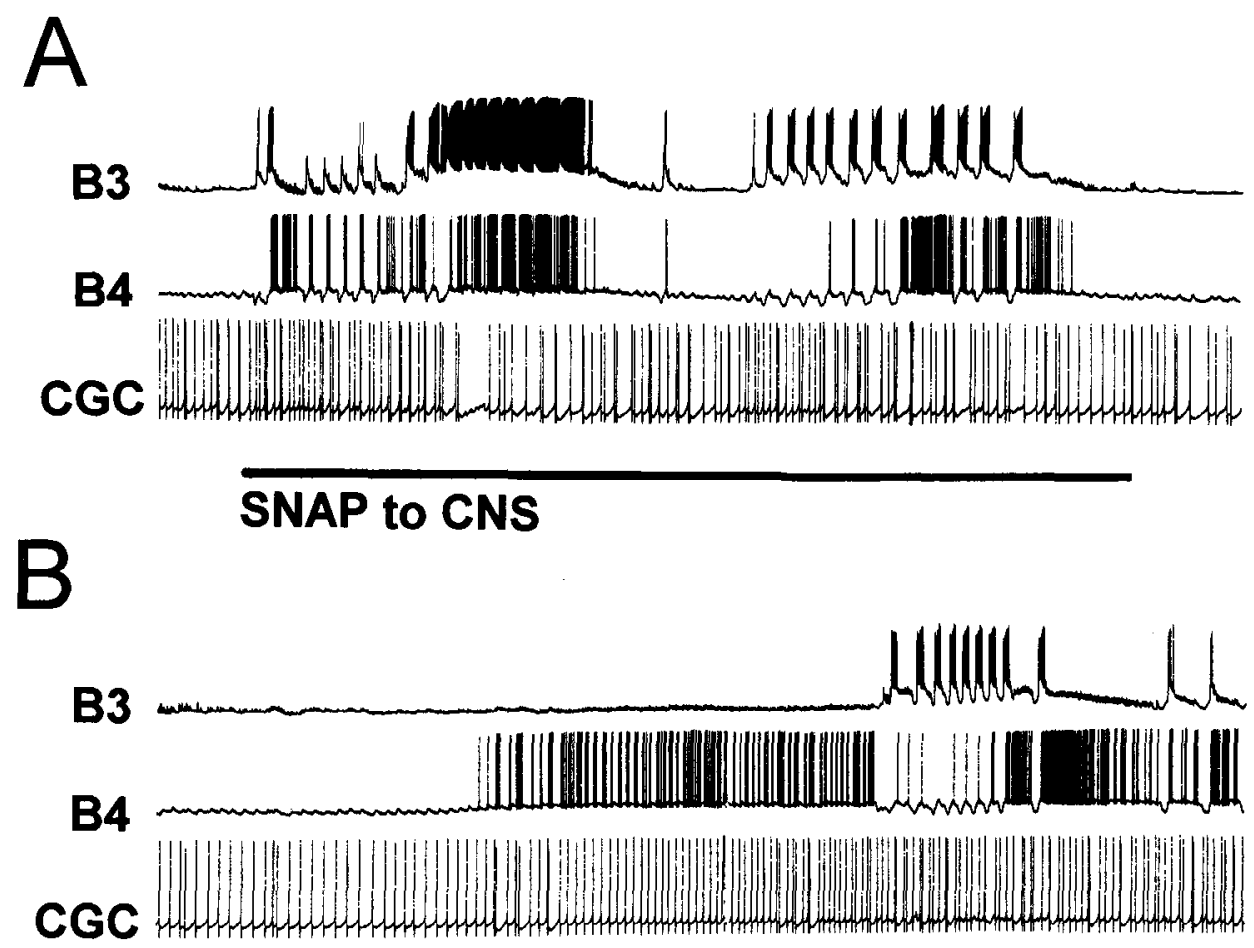

\section{HA to CNS}

Q

B1

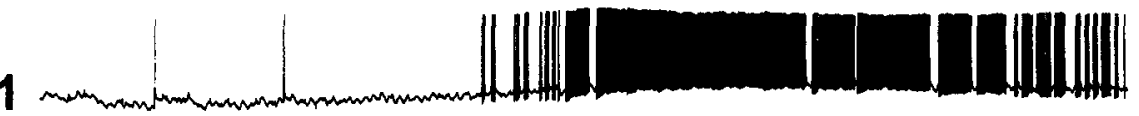

B4
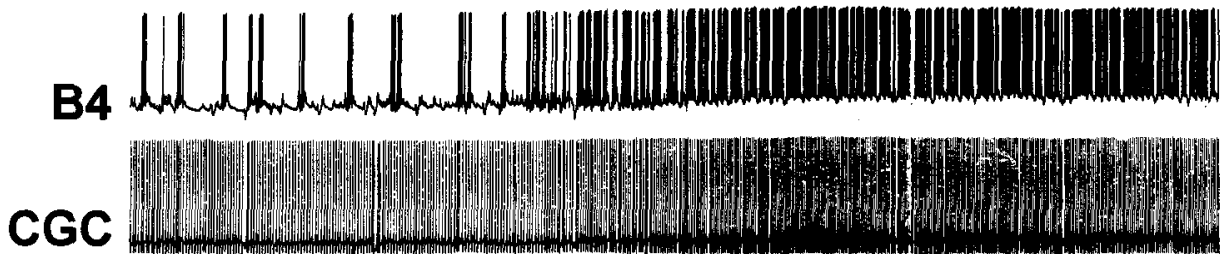

$50 \mathrm{mV}$

8-Br-cGMP to CNS

Figure 6. NO donors and 8-Bromo-cGMP (8-Br-cGMP) activate fictive feeding. $A$, The NO donor $S$-nitroso- $N$-acetylpenicillamine (SNAP, 1 mM) causes tonic excitation of the CGC and tonic depolarization and phasic activation of buccal motoneurons B3 and B4 (fictive feeding). $B$, Hydroxylamine $(H A ; 1 \mathrm{mM})$ also excites the CGC and activates fictive feeding but with a longer latency of onset than SNAP (see text for a possible explanation). For example, in this preparation the latency to the onset of fictive feeding, best seen in the B3 cell, is about 4 min. Note that prior to the onset of fictive fecding, tonic excitation can be scen in the B4 cell. C, 8-Br-cGMP ( $3 \mathrm{mM})$ induces tonic excitation of the modulatory CGC interneuron and both tonic depolarization and phasic activation of buccal motoneurons (fictive feeding). As with the NO donor hydroxylamine (B), the onset of fictive feeding activity is delayed compared with the response observed with SNAP $(A)$ or sucrose (Fig. $4 A$ ).

ration of the observation period. Hemoglobin caused complete suppression of feeding in all individuals tested throughout the entire observation period, in spite of appearing in all other respects to be behaving normally (Fig. 9A). As a control for nonspecific effects of protein injection, we tested animals following injection with Met-hemoglobin. In this control group, $40 \%$ of animals started feeding during the test period and the mean number of bites increased from $1.0 \pm 1.0$ (SEM) in the first $10 \mathrm{sec}$ interval to $5.3 \pm 0.7$ (SEM) in the last (Fig. 9A). The difference between the effects of hemoglobin and Met-hemoglobin are highly significant $\left(p<0.001, \chi^{2}\right.$ test) and while there is some inhibitory effect of Met-hemoglobin, we can attribute a major inhibitory effect to the NO-scavenging capacity of hemoglobin. The partial but rather strong inhibitory effect of Met-hemoglobin (compared to no treatment or distilled water treatment) suggested that a part of the very strong inhibitory effect of hemoglobin must be accounted for by actions unrelated to inhibition of NO signalling.

The time course of the population response to sucrose in the distilled water injected snails (control for methylene blue) was not different from that in the untreated animals (two-factor ANOVA with replicates $p=0.89$ ) (Fig. 9B). Methylene blue, on the other hand, caused a significant decrease in the number of rasps over the first $40 \mathrm{sec}$ of the observation period compared 
A

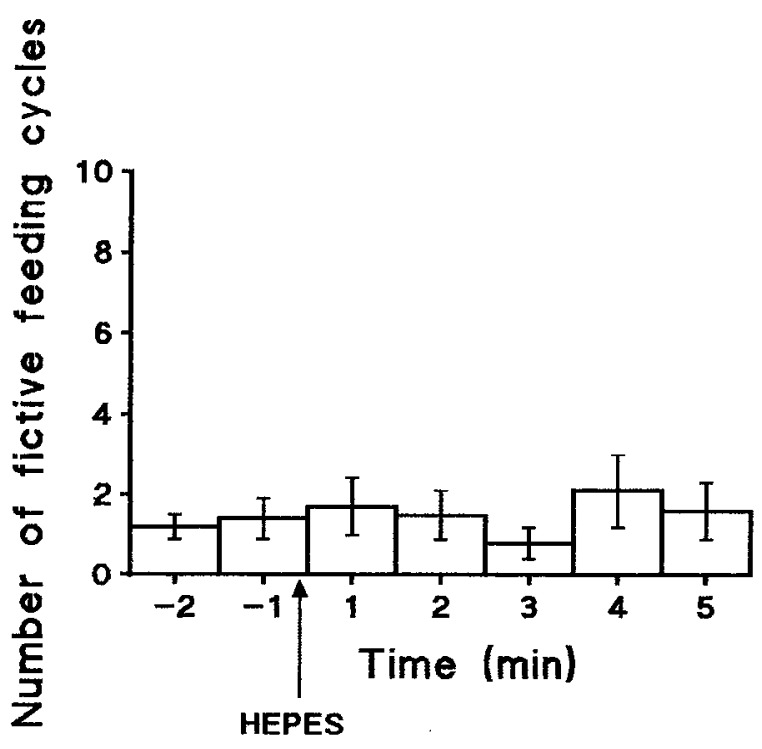

C

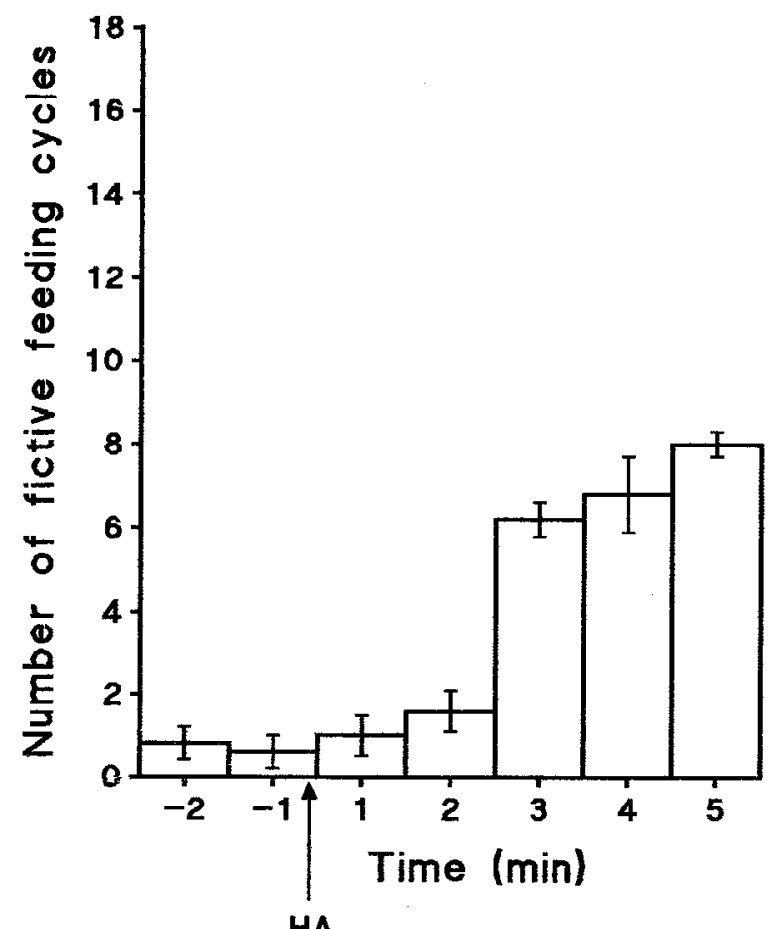

B
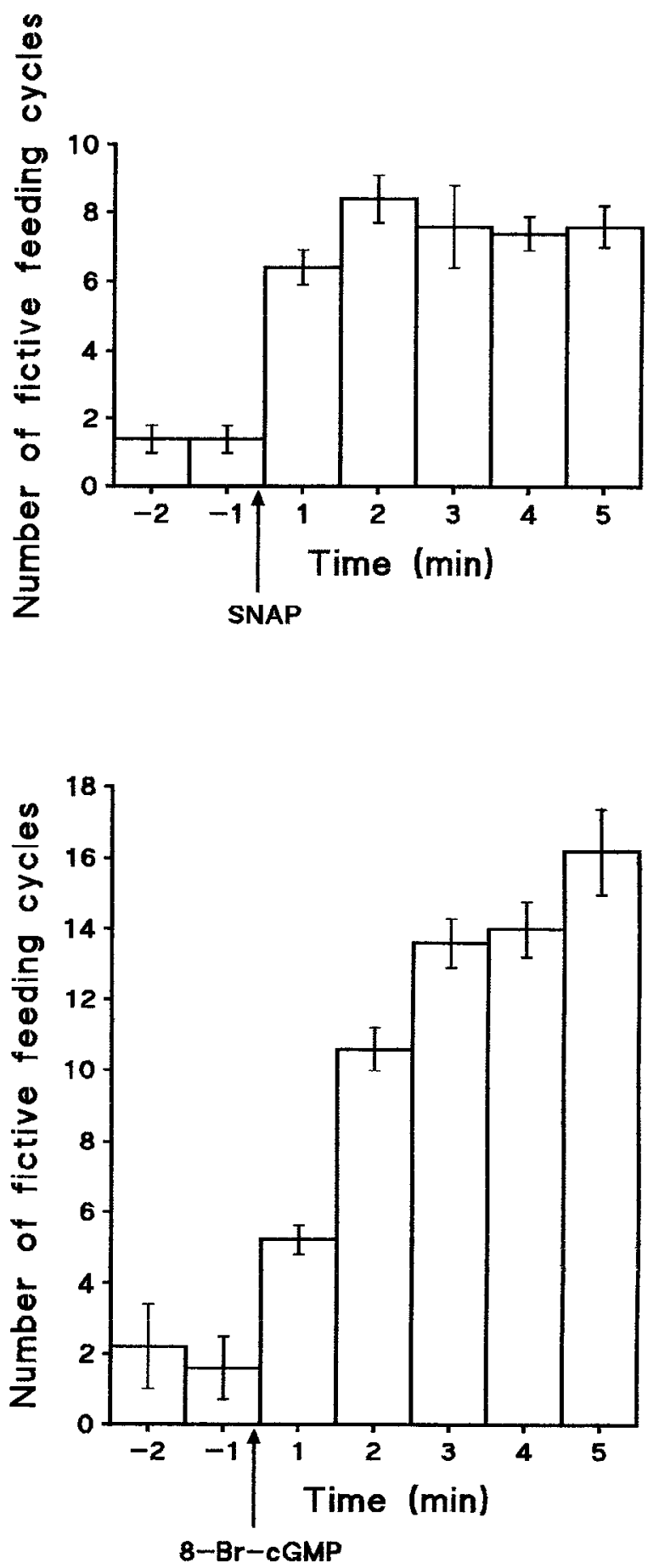

Figure 7. Analysis of NO donor and 8-Br-cGMP-induced fictive feeding. The graphs show the mean number of fictive feeding cycles \pm SEM ( $n$ $=5-10$ ) in each minute during the $2 \mathrm{~min}$ before and the $5 \mathrm{~min}$ after application of drugs to CNS. $A$, In controls (HEPES), no increase in the number of fictive feeding cycles is observed. $B$, SNAP $(1 \mathrm{mM})$ causes a rapid increase in the number of fictive feeding cycles. $C$, Hydroxylamine $(H A)$ causes a delayed increase in the number of fictive feeding cycles. $D, 8$-Br-cGMP causes a delayed increase in the number of fictive feeding cycles, which continues to rise over $5 \mathrm{~min}$ to rate of about $16 \mathrm{cycles} / \mathrm{min}$.

to both untreated and distilled water controls (two-factor ANOVA with replicates $p<0.001, t$ tests: $p<0.01$ to 0.05 ) (Fig. $9 B$ ). After the first $40 \mathrm{sec}$, however, there was no significant difference in the mean number of rasps between untreated, distilled water-treated and methylene blue-treated snails (two-factor
ANOVA with replicates $p=0.49$ ). This latter finding indicates a lack of effect of the inhibition of the NO-cGMP pathway on the general ability of the snails to generate the stereotyped behavioral pattern of feeding even when the response builds up more slowly. The differences found during the first $40 \mathrm{sec}$ of 


\section{A CNS in HEPES}

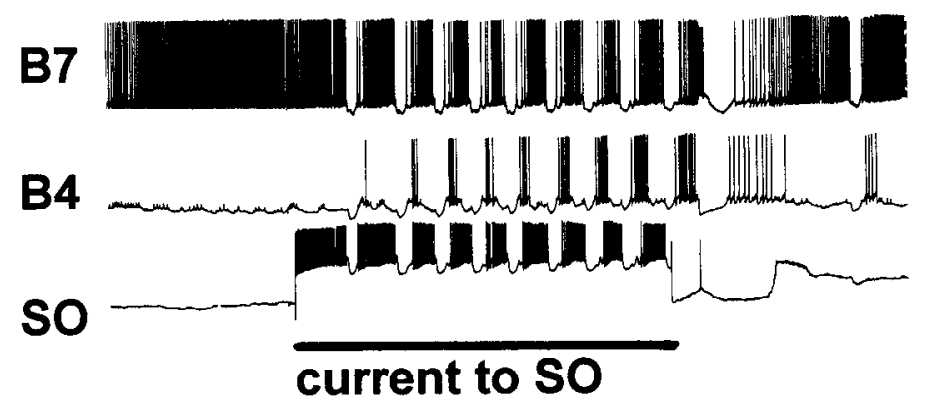

Figure 8. Hemoglobin does not inhibit centrally activated fictive feeding. $A$, Injection of depolarizing current into the modulatory SO interneuron triggers and maintains CPG-driven fictive feeding activity in buccal motoneurons. $B$, SO-activated fictive feeding is not affected by bathing the CNS with hemoglobin $(H b ; 0.2 \mathrm{mM})$, which is in contrast with the strong inhibitory effect of $\mathrm{Hb}$ on sucrose-induced fictive feeding (Fig. 4A).

B CNS in $\mathrm{Hb}$

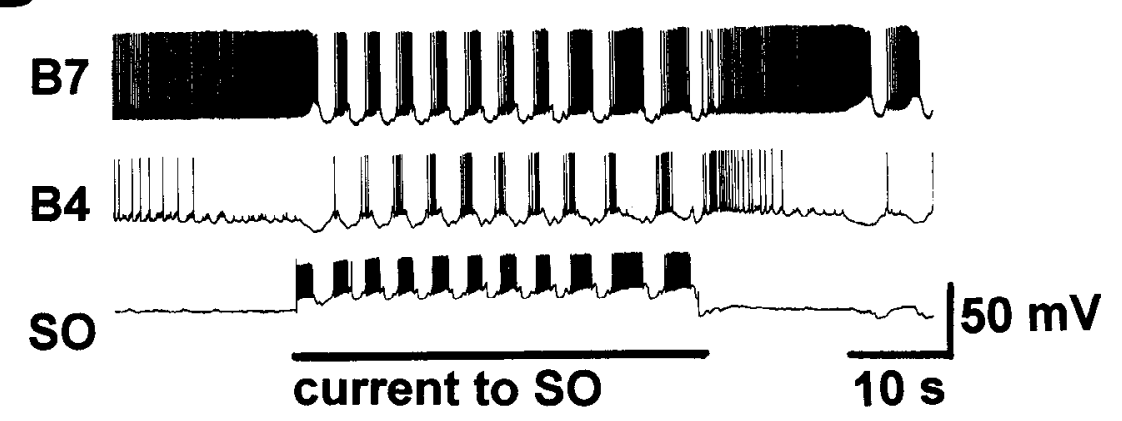

the observation period therefore indicate an inhibitory effect on the propagation of sensory information in methylene blue treated snails.

\section{Discussion}

The results of this study indicate a role for the NO-cGMP signalling pathway in mediating chemosensory activation of feeding behavior in the mollusc Lymnaea stagnalis. Our anatomical studies using NADPH diaphorase histochemistry show that there are putative NO-releasing chemosensory neurons in the lips that project to the cerebral ganglia. Intense NADPH diaphorase staining has also been observed in the lip sensory system of another pulmonate mollusc Helix aspersa (Cooke et al., 1994) and in the tentacular and lip nerves of Aplysia (Jacklet and Grohn, 1994a,b). In Helix, numerous NADPH diaphorase-positive sensory cells are present in the lips and tentacles and the tentacle and median lip nerves contain a large number of stained axons. These findings are very similar to ours, and suggest that in other molluscs the NO-cGMP signalling pathway may have a similar chemosensory role to that demonstrated here in Lymnaea.

Our experiments on semi-intact and intact snails indicate that $\mathrm{NO}$ has a behavioral role in mediating chemosensory activation of feeding. Electrophysiological experiments using a semi-intact lip-CNS preparation support this hypothesis. Activation of rhythmic activity in identified feeding motoneurons (fictive feeding) by sucrose applied to the lips was inhibited when the CNS was bathed with the NO scavenger hemoglobin or the NOS inhibitor L-NAME. Inhibition due to L-NAME is reversed by L-arginine, as demonstrated previously in Limax (Gelperin, 1994). Application of NO donors to the CNS mimicked sucrose-induced fictive feeding but central activation of fictive feeding by current injection into a feeding interneuron (SO) was unaffected by hemoglobin. Interestingly, these results indicate that NO is required for chemosensory activation of fictive feeding, but is not involved in transmission within the central network of neurons responsible for generating motor output.

Additional evidence was provided by experiments on the role of cGMP in chemosensory activation of feeding. For example, NO activates cGMP production in the Lymrnaea CNS, the guanylyl cyclase inhibitor methylene blue inhibits sucrose-induced fictive feeding and application of the cGMP analog 8-BromocGMP to the CNS mimics sucrose-induced fictive feeding. Methylene blue is known to be an inhibitor of NO-activated guanylyl cyclase (Miki et al., 1987) and may also inhibit NOS (Mayer et al., 1993). It is possible, however, in addition that methylene blue has nonspecific effects in the nervous system that could account for the inhibitory effects of methylene blue that we have observed. In order to examine this possibility we investigated the effect of methylene blue on synaptic transmission between identified neurons in the feeding circuitry. No ef fects on the CGC to motoneuron or CPG to motoneuron connections were observed, which supports our conclusion that the inhibitory effect of methylene blue on sucrose-activated fictive feeding is specific and can be attributed to inhibition of the NOcGMP pathway. In Aplysia, methylene blue has been used selectively to block the synaptic depolarization of a follower neuron by activation of a diaphorase positive cerebral neuron (Jacklet and Gruhn, 1994b).

Direct behavioral experiments on intact animals also support the conclusions from fictive feeding experiments. Thus, injection of the NO scavenger hemoglobin inhibited a sterentypical feeding response of hungry snails in sucrose-rich water and methylene blue caused a significant delay in the onset of feeding compared to controls. The rate of rasping where feeding was initiated in treated animals, however, was the same as in untreated animals, indicating that feeding behavior could be performed normally in the treated animals but was not activated by sucrose in 
A

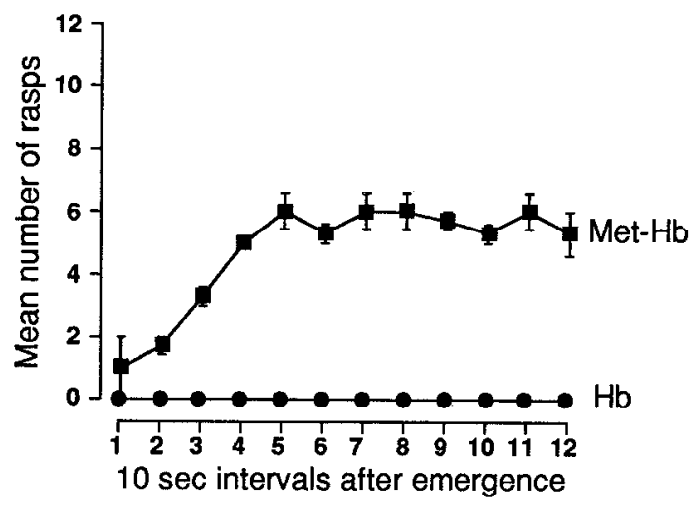

B

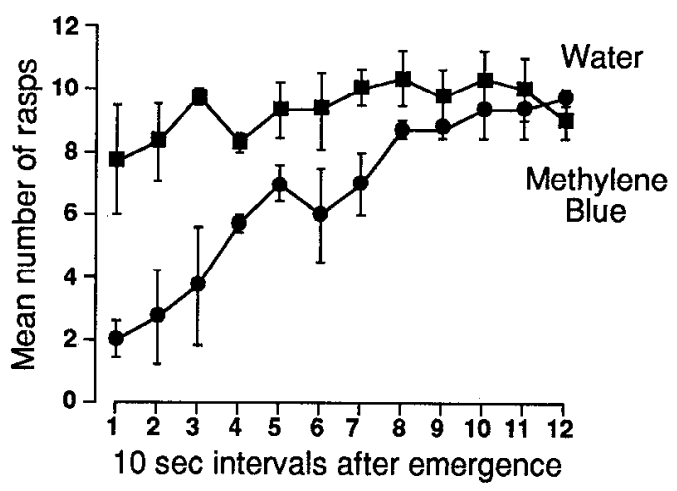

Figure 9. Effect of NO-cGMP pathway inhibitors on sucrose-induced feeding behavior. The graphs compare the feeding response to sucrose of snails injected with: $A$, the NO scavenger hemoglobin $(H b)$ or with Met-hemoglobin $($ Met-Hb) as a control, and $B$, the guanylyl cyclase inhibitor methylene blue or water (control). The graphs show the mean ( \pm SEM, $n=3$ ) number of rasps in a group of four animals in each $10 \mathrm{sec}$ interval of a 2 min period following emergence in sucruse-rich water. In the Met-Hb group there is a gradual increase to a maximal rasping rate of about six per $10 \mathrm{sec}$ during the first minute after emergence, which is then sustained. In contrast, in the Hb group no rasping was observed in any individuals for the duration of the observation period. In the water-injected group there was a rapid onset of rasping to a near maximal rasping rate during the first $10 \mathrm{sec}$ interval after emergence. In contrast, in the methylene blue group there was a significantly slower onset of rasping and a maximal rate of rasping was not achieved until the eighth $10 \mathrm{sec}$ interval.

the normal way. The delayed activation may be caused by the action of sucrose on receptors in the buccal cavily (Kemenes et al., 1986) that do not use the NO signalling pathway. These results corroborate our anatomical and in vitro findings, indicating that the NO-cGMP pathway mediates chemosensory activation of feeding but not central control or organization of this behavior. The difference between the waning of the $\mathrm{Hb}$ and $\mathrm{MB}$ effects may be due to the presence of an additional large protein molecule in the blood, which at high concentration leads to a behaviural state resembling food satiety.

The results of our behavioral experiments with hemoglobin might suggest that naturally circulating oxygen-binding proteins in the hemolymph influence chemosensory activation of feeding in Lymnaea. The principal oxygen carrying molecule in Lymnaea is hemocyanin, but importantly, the circulating concentration of this molecule $\left(<10^{-5} \mathrm{M}\right.$; Pullin, 1971) is about one hundred times lower than the estimated hemolymph concentration of injected hemoglobin in our behavioral experiments. Moreover, molecular mass of hemocyanin is between 3 and 7.5 million Da, which confines it to the circulation (Schindelmeiser et al., 1979), whereas the $64 \mathrm{kDa}$ hemoglobin can gain access to the CNS. We therefore consider it unlikely that oxygen-binding proteins in Lymnaea hemolymph influence feeding pharmacologically.

The results and conclusions of this study are also supported by a number of other recent studies. Moroz et al. (1993) observed that the NO donor S-nitrosocysteine activates feeding movements of the buccal mass and modulates the activity of buccal motoneurons in Lymnaea stagnalis. These authors concluded that the natural source of NO in the Lymnaea feeding system might be the population of NADPH diaphorase-positive neurons in the buccal ganglion (Elofsson et al., 1993; Moroz et al., 1993; Elphick et al., 1994b), which are also immunoreactive with an antiserum to rat cerebellar NOS (Moroz et al., 1994). Although these putative and so far unidentified NO-releasing buccal neurons may influence the activity of feeding motoneurons, our findings indicate that the principal source of NO in the cerebral compartment of the feeding system are the centrally projecting NADPH diaphorase-positive sensory cells in the lips.

Together, our findings indicate that NO is released in the CNS of Lymnaea by NOS-containing lip chemosensory neurons where it activates feeding behavior by stimulating cGMP production in neurons of the central feeding network. Evidence for a role for $\mathrm{NO}$ as a transmitter of an identified sensory neuron in a mollusc has recently been reported (Jacklet, I995). In this example, NO appears to be coreleased with histamine from the $\mathrm{C} 2$ neuron of the cerebral ganglion of Aplysia. The behavioral significance of this has, however, yet to be determined. Specific target neurons for NO in the feeding network in Lymnaea are not yet known but likely candidates include the modulatory serotonergic CGC interneurons and other identified modulatory or CPG feeding interneurons. These uniquely identifiable neurons provide a model system in which the cellular actions of NO can be understood comprehensively in a behavioral context.

\section{References}

Benjamin PR, Elliott CJH (1989) Snail feeding oscillator: the central pattern generator and its control by modulatory interneurons. In: Neuronal and cellular oscillators (Jacklet JW, ed), pp 173-174. New York: Dekker.

Benjamin PR, Rose RM (1979) Cental generation of bursting in the feeding system of the snail Lymnaea stagnalis. J Exp Biol 80:93118.

Benjamin PR, Winlow W (1981) The distribution of three wide-acting inputs to identified neurons in the isolated brain of Lymnaea stagnalis. Comp Biochem Physiol 70A:293--307.

Bredt DS, Snyder SH (1989) Nitric oxide mediates glutamate-linked enhancement of cGMP levels in the cerebellum. Proc Natl Acad Sci USA 86:9030-9033.

Bredt DS, Snyder SH (1992) Nitric oxide, a novel neuronal messenger. Neuron 8:3-11.

Cooke IRC, Edwards SL, Anderson CR (1994) The distribution of NADPH diaphorase activity and immunoreactivity to nitric oxide synthase in the nervous system of the pulmonate mollusc Helix aspersa. Cell Tissue Res 277:565-572.

Dawson TM, Bredt DS, Fotuhi M, Hwang PM, Snyder SH (1991) Nitric oxide synthase and neuronal NADPH diaphorase are identical in brain and peripheral tissues. Proc Natl Acad Sci USA 88:7797-7801.

Elliott CJH, Benjamin PR (1985a) Interactions of pattern generating interneurons controlling feeding in Lymnaea stagnalis. J Neurophysiol 54:1396-1411.

Elliott C.IH, Benjamin PR (1985b) Interactions of the slow oscillator interneuron with feeding pattern generating interneurons in Lymnaea stagnalis. J Neurophysiol 54:1412-1421. 
Elphick MR, Grecn IC, O'Shca M (1993) Nitric oxide synthesis and action in an invertebrate brain. Brain Res 619:344-346.

Elphick MR, Green IC, O'Shea M (1994a) Nitric oxide signalling in the insect nervous system. In: Insect neurochemistry and neurophysiology 1993 (Borkovek AB, Loeb M, eds), pp 129-132. Boca Raton, FL: CRC.

Elphick MR, Riveros-Moreno V, Moncada S, O'Shea M (1994b) Identification of nitrergic neurons in invertebrates. In: The biology of nitric oxide: physiology and clinical aspects, Vol 3 (Moncada S, Feelisch M, Busse R, Higgs EA, eds), pp 377-381. London: Portland.

Elphick MR, Rayne RC, Riveros-Moreno V, Moncada S, O'Shea M (1995) Nitric oxide synthesis in locust olfactory interneurons. J Exp Biol 198:821-829.

Elofsson R, Carlberg M, Moroz L, Nezlin L, Sakharov D (1993) Is nitric oxide (NO) produced by invertebrate neurones? Neuroreport 4:279-282

Feelisch M (1991) The biochemical properties of nitric oxide formation from nitrovasodilators: appropriate choices of exogenous NO donors and aspects of preparation and handling of aqueous NO solutions. $J$ Cardiovasc Pharmacol 17(Suppl 3):S25-S33.

Gelperin A (1994) Nitric oxide mediates network oscillations of olfactory interneurons in terrestrial mollusc. Nature 369:61-63.

Goldschmeding JT, Jager JC (1973) Feeding responses to sucrose in the pond snail Lymnaea stagnalis after nerve section and tentacle amputation. Neth J Zool 23:118-124.

Hope BT, Michael GJ, Knigge KM, Vincent SR (1991) Neuronal NADPH diaphorase is a nitric oxide synthase. Proc Natl Acad Sci USA 88:2811-2814.

Jacklet JW (1995) Nitric oxide is used as an orthograde cotransmitter at identified histaminergic synapses. J Neurophysiol, in press.

Jacklet JW, Gruhn M (1994a) Co-localisation of NADPH-diaphorase and myomodulin in synaptic glomeruli of Aplysia. Neuroreport 5:1841-1844.

Jacklet JW, Gruhn M (1994b) Nitric oxide as a putative transmitter in Aplysia neural circuits and membrane effects. Neth J Zool 44:524534.

Kemenes G, Elliott CJH, Benjamin PR (1986) Chemical and tactile inputs to the Lymnaea feeding system: effects on behavior and neural circuitry. J Exp Biol 122:113-137.

Kemenes G, Elliott CJH (1994) Analysis of the feeding motor pattern in the pond snail Lymnaea stagnalis: photoinactivation of axonally stained pattern generating interneurons. J Neurosci 14:153-166.

Lambert LE, Whitten JP, Baron BM, Cheng HC, Doherty NS, McDonald IA (1990) Nitric oxide synthesis in the CNS, endothelium and marcophages differs in its sensitivity to inhibition by arginine analogues. Life Sci 48:69-75.

Mayer B, Brunner F, Schmidt K (1993) Inhibition of nitric oxide synthesis by methylene blue. Biochem Pharmacol 45:367-374.

McCrohan CR, Benjamin PR (1980a) Patterns of activity and axonal projections of the cerebral giant cells of the snail, Lymnaea stagnalis. J Exp Biol 85:149-168.

McCrohan CR, Benjamin PR (1980b) Synaptic relationships of the cerebral giant cells with motoneurons in the feeding system of Lymnaea stagnalis. J Exp Biol 85:169-186.

Miki N, Kawabe V, Kuriyama K (1987) Activation of cerebral guanylate cyclase by nitric oxide. Biochem Biophys Res Commun 75: 851-856.

Moncada S, Palmer RMJ, Higgs EA (1991) Nitric oxide-physiology, pathophysiology and pharmacology. Pharmacol Rev 43:109-142.

Moriguchi M, Manning LR, Manning JM (1992) Nitric oxide can modify amino acid residues in proteins. Biochem Biophys Res Commun 183:598-604.

Moroz L, Park J-H, Winlow W (1993) Nitric oxide activates buccal motor patterns in Lymnaea stagnalis. Neuroreport 4:643-646.

Moroz LL, Winlow W, Turner RW, Bulloch AGM, Lukowiak K, Syed NI (1994) Nitric oxide synthase-immunoreactive cells in the CNS and periphery of Lymnaea. Neuroreport 5:1277-1280.

Pape H-C, Mager R (1992) Nitric oxide controls oscillatory activity in thalamocortical neurons. Neuron $9: 441-448$.

Pullin RSV (1971) Composition of the heamolymph of Lymnaea truncatula, the snail host of Fasciola hepatica. Comp Biochem Physiol 40A:617-626.

Rose RM, Benjamin PR (1979) The relationship of the central motor pattern to the feeding cycle of Lymnaea stagnalis. J Exp Biol 80: $137-163$.

Rose RM, Benjamin PR (1981a) Interneuronal control of feeding in the pond snail, Lymnaea stagnalis. I. Initiation of feeding cycles by a single buccal interneuron. J Exp Biol 92: 187-201.

Rose RM, Benjamin PR (1981b) Interneuronal control of feeding in the pond snail, Lymnaea stagnalis. II. The interneuronal mechanism generating feeding cycles. J Exp Biol 92: 203-228.

Schindelmeiser I, Kuhlmann D, Nolte A (1979) Localization and characterization of hemoproteins in the central nervous tissue of some gastropods. Comp Biochem Physiol 64B:149-154.

Schuman EM, Madisson DV (1994) Nitric oxide and synaptic function. Annu Rev Neurosci 17:153-183.

Southam E, Garthwaite J (1991) Comparative effects of some nitric oxide donors on cyclic GMP levels in rat cerebellar slices. Neurosci Lett 130:107-111.

Southam E, Garthwaite J (1993) The nitric oxide-cyclic GMP signalling pathway in rat brain. Neuropharmacology 32:1267-1277.

Yeoman MS, Pieneman AW, Ferguson GP, Ter Maat A, Benjamin PR (1994a) Modulatory role for the serotonergic Cerebral Giant Cells in the feeding system of the snail, Lymnaea. I. Fine wire recording in the intact animal and pharmacology. J Neurophysiol 72: 1357-1371.

Yeoman MS, Kemenes G, Benjamin PR, CJH Elliott (1994b) Modulatory role for the serotonergic Cerebral Giant Cells in the feeding system of the snail, Lymnaea. II. Photoinactivation. J Neurophysiol 72:1372-1382.

Zaitseva OV, Bocharova LS (1981) Sensory cells in the head skin of pond snails. Cell Tissue Res 220:797-807. 